\title{
Erratum to: Development of Zeldovich's approach for cosmological distances measurement in the Friedmann Universe
}

\author{
A. V. Nikolaev ${ }^{1, a}$, S. V. Chervon ${ }^{1,2, b}$ \\ ${ }^{1}$ Ilya Ulyanov State Pedagogical University, 100 years of V.I. Lenin's Birthday Square B. 4, 432700 Ulyanovsk, Russia \\ ${ }^{2}$ Astrophysics and Cosmology Research Unit, School of Mathematics, Statistics and Computer Science, University of KwaZulu-Natal, \\ Private Bag, X54 001, Durban 4000, South Africa
}

Received: 22 June 2017 / Published online: 4 July 2017

C) The Author(s) 2017. This article is an open access publication

\section{Erratum to: Eur. Phys. J. C (2015) 75:411 DOI 10.1140/epjc/s10052-015-3614-3}

The present remarks as regards Ref. [1] result from a useful discussion with Helbig, one of the authors of Ref. [2]. We would like to clarify this in what follows.

In Ref. [1] we derived the generalized differential equation (32) using such tools as null geodesics and the ratio of longitudinal to transverse angular momentum of a photon. We also repeated calculations using the focusing equation [3], where we proved that the general form of the equation for the angular diameter distance in $\Lambda C D M$ is

$\ddot{d}_{\mathrm{a}}-\frac{\dot{a}}{a} \dot{d}_{\mathrm{a}}+4 \alpha \pi G(p+\rho) d_{\mathrm{a}}=0$.

Equation (1) is the generalised version of the Dashevskii equation in [4] for a model with the equation of state for several components with arbitrary equations of state. (Note that in this form, it is assumed that all of these components clump in the same manner.) Displaying the components for the $\Lambda C D M$ model we obtain

$p+\rho=p_{\Lambda}+p_{M}+p_{R}+\rho_{\Lambda}+\rho_{M}+\rho_{R}$.

Using the equations of state for baryonic matter $\left(p_{M}=0\right)$, dark energy $\left(p_{\Lambda}=-\rho_{\Lambda}\right)$, and radiation $\left(p_{R}=\rho_{R} / 3\right)$, we convert Eq. (2) to

The online version of the original article can be found under doi:10.1140/epjc/s10052-015-3614-3.

\footnotetext{
a e-mail: ilc@xhns.org

b e-mail: chervon.sergey@gmail.com
}

$p+\rho=\frac{4}{3} \rho_{R}+\rho_{M}$

Using (3), Eq. (1) acquires the form

$\ddot{d}_{\mathrm{a}}-\frac{\dot{a}}{a} \dot{d}_{\mathrm{a}}+4 \alpha \pi G\left(\frac{4}{3} \rho_{R}+\rho_{M}\right) d_{\mathrm{a}}=0$.

Then if we set $\rho_{R}=0$ we will obtain the Dashevskii equation in [4]. This result was not known to us at the time when we were writing Ref. [1]; that is why we wrote that the authors [2] obtained a wrong result. The authors of [2] started from the old Dashevskii equation, but not from (4). This means that the equation obtained by Dashevskii for the model without $\Lambda$ (or dark energy) term is still valid in $\Lambda C D M$, which was not obvious.

Because in such a setup the Dashevskii equation remains valid in $\triangle C D M$, the final equation for the ADD measurement is true. Nevertheless we should note that it is unnecessary to use the equation from [2], because it is much simpler to integrate the Dashevskii equation itself, after converting the redshift to cosmic time, as was shown in Ref. [1].

Thus we can summarize as follows:

- The more general version of the differential equation for the angular diameter distance for models containing components with arbitrary equations of state is our result Eq. (1).

- If the dark energy equation of state is $p_{\Lambda}=-\rho_{\Lambda}$ and $\rho_{R}=0$, which corresponds to the cosmological constant, then the original Dashevskii equation is valid for $\Lambda C D M$. This explains why Ref. [2] produces valid results.

- The code presented in [2] produces correct results. 
Open Access This article is distributed under the terms of the Creative Commons Attribution 4.0 International License (http://creativecomm ons.org/licenses/by/4.0/), which permits unrestricted use, distribution, and reproduction in any medium, provided you give appropriate credit to the original author(s) and the source, provide a link to the Creative Commons license, and indicate if changes were made.

Funded by SCOAP ${ }^{3}$.

\section{References}

1. A.V. Nikolaev, S.V. Chervon, EPJC 75, 411 (2015)

2. R. Kayser, P. Helbig, T. Schramm, Astron. Astrophys. 318, 680 (1997)

3. A. Nikolaev, S. Chervon, Gravit. Cosmol. 22, 208 (2016)

4. V.M. Dashevskii, V.I. Slysh, Sov. A 9, 671 (1965) 